\title{
Regressive melanoma in a female patient: A case report
}

\author{
FLORICA SANDRU ${ }^{1,2}$, CARMEN-CRISTINA DRAGHICI $^{2}$, TEODORA PREDESCU ${ }^{3}$, \\ MARIA MAGDALENA CONSTANTIN ${ }^{1,4}$, RĂZVAN-COSMIN PETCA ${ }^{1,5}$, TRAIAN CONSTANTIN ${ }^{5}$, \\ AIDA PETCA $^{1,6}$ and MIHAI CRISTIAN DUMITRAȘCU ${ }^{1,7}$
}

\author{
${ }^{1}$ 'Carol Davila' University of Medicine and Pharmacy, 050474 Bucharest; ${ }^{2}$ Department of Dermatology, \\ 'Elias' Emergency University Hospital, 011461 Bucharest; ${ }^{3}$ Department of Dermatology, Bio Terra Med Clinic, \\ 014192 Bucharest; ${ }^{4}$ Department of Dermatology II, Colentina Clinical Hospital, 020125 Bucharest; ${ }^{5}$ Department of Urology, \\ 'Prof. Dr. Theodor Burghele' Clinical Hospital, 061344 Bucharest; ${ }^{6}$ Department of Obstetrics and Gynecology, \\ 'Elias' Emergency Hospital, 011461 Bucharest; ${ }^{7}$ Department of Obstetrics and Gynecology, \\ University and Emergency Hospital Bucharest, 050098 Bucharest, Romania
}

Received December 18,2019; Accepted January 17, 2020

DOI: $10.3892 /$ etm.2020.8675

\begin{abstract}
Regressive melanoma is a phenomenon characterized by partial or complete replacement of cutaneous melanoma by fibrotic structures as a result of local host immune response. The dermoscopic features include 'scar-like' depigmentation that can be described as hypopigmented to pigmented macules, pink macules, linear-irregular vessels, globular vessel pattern, hyperpigmented macular remnants, blue gray 'peppered' papular remnants and white transverse bands. Fully regressive melanoma is very rare, and, in majority of cases, the tumor is associated with metastases, so the prognosis is very poor due to this fact. Regressive melanomas are rare since it represents an advance stage of melanoma evolution. Their treatment requires a collaboration between the dermatologist and the oncologist in order to improve the quality of life of the patient and also the survival rate.
\end{abstract}

\section{Introduction}

Melanoma is a malignant tumor that derives from melanocytes found in the basal layer of the epidermis but also in other organs such as oral and nasal mucosa, uvea, vulvar and anorectal mucosa, brain or gastrointestinal mucosa. Skin is the commonest site of involvement of melanoma. Cutaneous

Correspondence to: Dr Carmen-Cristina Draghici, Department of Dermatology, 'Elias' Emergency University Hospital, Bulevardul Mărăști 17, 011461 Bucharest, Romania

E-mail: carmen.draghici3@gmail.com

Abbreviations: UV, ultraviolet; $\mathrm{MxA}$, myxovirus resistance A; CXCR3, chemokine receptor; IP10/CXCL10, interferon- $\gamma$-induced protein/C-X-C motif chemokine 10; CD68, cluster of differentiation 68; ICAM-1, intercellular adhesion molecule-1

Key words: regressive melanoma, metastasis, prognostic melanoma is the most aggressive skin cancer and a major cause of premature death.

Based on epidemiologic studies, melanoma is the sixth most common fatal malignancy in USA and the second most common cancer in women aged between 20 and 29 years. Moreover, it is considered that, in the future, almost one million of new melanomas will be diagnosed every year in USA (1-3).

Melanoma risk factors include ultraviolet light radiation from the sunlight but also from artificial sources of lights such as tanning beds, history of sunburns in childhood or adolescence, the number of congenital and acquired nevi and genetic susceptibility or a family history of melanoma. Regarding ultraviolet light radiation, melanoma developing risk is associated with UV level and particularly UV-B, in case of sunlight exposure or UV-A artificial light (3-6).

Regressive melanoma is a phenomenon characterized by the partial or complete replacement of cutaneous melanoma by fibrotic structures as a result of local host immune response (7).

Cutaneous melanoma can be clinically described as an asymmetric skin lesion with irregular border and color variation from brown, dark brown, black or blue while the regression phenomenon can be initially subtle and even unnoticed evolving from hyperpigmentation to depigmentation of one or multiple parts of the lesion resulting in blue, pink, white or gray areas.

Regarding the histologic criteria there is still no standard definition of histopathologic aspects in regression of primary melanoma. The histologic findings include variably dense mononuclear infiltrate, melanophages, dermal fibrosis, epidermal attenuation and dense vascularity in dermis. These histologic changes are also seen in the absence of clinical regression $(8,9)$.

Some authors have classified regression in three stages: Early stage of regression characterized by mononuclear inflammatory cell infiltrate with mature lymphocytes and keratinocyte and disruption of the dermal layer; the intermediate stage with near complete absence of melanocytes, the presence of melanophages, lymphohistiocytic infiltrate, fibroblastic 
proliferation, mild collagen deposition and increased vascular pattern in the superficial dermal layer; and the late stage described as a lesion with complete absence of melanocytes, fibrosis in the superficial/papillary dermis, ectatic capillaries, variable numbers of melanophages and a small number of inflammatory cells (10).

A rare case of regressive melanoma in a 62-year-old female patient with no other general symptoms before the admission to our clinic is presented.

\section{Case report}

We present the case of a 62-year-old female patient who was referred to our clinic with a depigmented skin lesion with areas of brown/black pigment in the middle, located on the left cheek. The patient could not tell when the lesion first appeared, but she confirmed the presence of a nodular hyperpigmented lesion on the left cheek that started to bleed two years ago after a local trauma. The depigmentation started three months earlier.

The clinical examination revealed a cutaneous $7 / 2 \mathrm{~cm}$ asymmetric patch with large pink discolorations and a central nodular, fibrotic pigmented area (Fig. 1). No ulceration, crusting or active bleeding was seen.

The general examination revealed multiple cervical lymphadenopathy with the largest one measuring $\sim 3 / 2 \mathrm{~cm}$. The lymph nodes were non-tender, firm in consistency, mobile and some were matted together. Skin in the cervical area was normal, with no sign of infiltration, inflammation or infection. No other lymph nodes were detected by clinical examination.

The dermoscopic examination revealed a massive depigmentation and a central dark black pigmented area with blue veil and scar-like depigmentation, and multiple dark brown 'pepper-like' dots in the middle of this structure (Fig. 2).

Due to its significant extension, it was decided to perform a skin biopsy from the middle of the lesion in order to encompass both pink depigmented area and dark-brown pigmented skin.

The histologic examination revealed infiltrated nodular dermal tissue with intense pigmented melanophages, dermal fibrosis, vascular hyperplasia and flat epidermal layer. The histopathologic changes correspond to the diagnosis of regressive melanoma. The immunohistochemistry tests with Giemsa counterstain on formalin fixed, paraffin embedded tissue sections were negative for Melan A and Tyrosinase presence in dermal cellular infiltrate and positive for S-100 in dispersed dendritic cells. It was also observed that D2-40 was positive in the lymphatic endothelium and did not show the presence of lymphatic tumor emboli while CD68 was positive in dermal cellular infiltrate. The data supported the diagnosis of regressive melanoma.

CT examination was performed revealing multiple lymph node metastases together with lung and brain metastases. The brain metastases were located supratentorial in both cerebral hemispheres with axial diameter of $46 / 37 \mathrm{~mm}$ in right fronto-parietal area and $36 / 35 \mathrm{~mm}$ in left frontal area, being spontaneous and hyperdense (probably a bleeding process). No bones metastases were seen.

Due to the advance stage of the disease, the patient was admitted to the oncological department for palliative treatment.

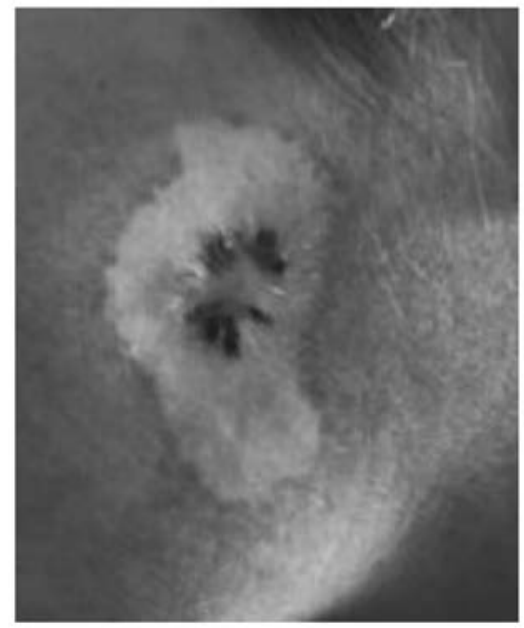

Figure 1. Cutaneous asymmetric hypopigmented patch with a central nodular and fibrotic pigmented area.

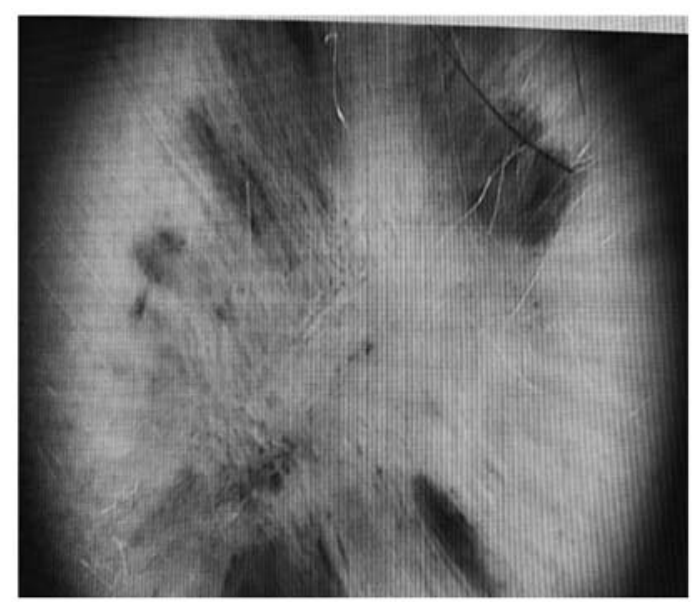

Figure 2. Massive depigmentation and a central dark black pigmented area with blue veil and scar-like depigmentation, multiple dark brown 'pepper-like' dots in the middle of this structure.

\section{Discussion}

Regression is a phenomenon that occurs as a result of host immune response that attacks the primary melanocytic tumor cells through lymphocytes determining a fibrotic process. The mechanism of regression occurs in 35\% of skin melanomas. Few cases of fully regressive melanomas are described in the literature. Regarding the metastatic sites, brain is the commonest with an incidence of $91 \%$ in cutaneous melanoma due to the capacity of tumoral cells to migrate along the external surfaces of vascular channels without extravasation (11). The majority of regressive melanomas were described after the occurrence of metastases, being one of the reasons that regression is considered to be a negative prognostic factor in melanoma (12).

There are some clinical studies that observed the immunological mechanism implied in regressive phenomenon. It is well known that cytotoxic T-cell response has an integral role in the regression process of melanomas. Other molecules were also identified in this process. A study outlined the high expression of MxA which was found in inflamed melanoma. 
MxA is an antiviral protein induced by type I interferon. Interferon also induces chemokine IP10/CXCL10, CXCR ${ }^{+}$ lymphocyte and granzyme $\mathrm{B}^{+}$lymphocytes. Collectively this evidence demonstrated that cytotoxic immune response is implied in spontaneous regression of melanoma (13). Another study showed the involvement of cytotoxic immune response through the correlation between expression of ICAM-1 and $\mathrm{CD}^{+}$cells near tumor cells (14). The role of interferon is sustained by the studies that confirmed the appearance of regressive changes of melanomas after the treatment with $\alpha$ interferon (15-18).

Regarding the dermoscopic changes in regressive melanoma, Bories et al (19) proposed seven criteria: 'Scar-like' depigmentation that can be described as hypopigmented to pigmented macules, pink macules, linear-irregular vessels, globular vessel pattern, hyperpigmented macular remnants, blue-gray 'peppered' papular remnants and white transverse bands.

The diagnosis of regressive melanoma is the same as for primary cutaneous melanoma and set after histologic examination on hematoxylin and eosin staining (20). Immunohistochemical test can also help differentiate between benign and malignant tumor.

The management of regressive melanoma depends on Breslow's thickness (the length from the granulosum stratum of epidermis to the deepest margin of the tumor), number of mitosis per square millimeter and ulceration, all helping to establish the tumor stage and appropriate therapeutic management.

The authors consider that patient with fully or partially regressive cutaneous melanoma should have the same diagnosis and treatment management as primary cutaneous melanoma (21-25).

In conclusion, regressive process of melanoma is a rare phenomenon that is usually noticed after the onset of visceral metastases. The immune mechanism is unclear but is considered that cytotoxic T-cell response is part of the regressive process. The prognosis of regressive melanomas is poor, mainly because most of them are associated with lymph nodes or organ metastases. We outlined this case because the patient was referred to dermatology unit due to the pink macule and not for general symptoms. The general examination showed the advance stage of the disease due to numerous cervical adenopathy and their tendency towards confluence. The brain CT showed cerebral metastases, so no curative treatment could be offered.

\section{Acknowledgements}

Not applicable.

\section{Funding}

Not funding was received.

\section{Availability of data and materials}

The datasets used and analyzed during the current study are available from the corresponding author on reasonable request.

\section{Authors's contributions}

AP contributed to the design of the case study. FS was involved in the writing of the manuscript and was responsible for the patient's follow up based on clinical and imaging examinations. CCD was responsible for the figures and was also involved in the conception of the study. TC analyzed the data from the literature research regarding regressive melanoma, and contributed in the writing of the manuscript. TP examined the patient and contributed in the writing of the manuscript regarding the clinical and dermoscopic examination of the lesion. MMC analyzed the histologic characteristics of regression and was responsible for the writing of the relevant section. RCP analyzed the melanoma risk factors and wrote the relevant section of the manuscript. MCD was also involved in the conception and design of the study, and contributed in the writing of the manuscript. All authors critically revised the manuscript and approved the final version of the manuscript to be published.

\section{Ethics approval and consent to participate}

Written informed consent was provided by the patient.

\section{Patient consent for publication}

The patient provided written informed consent for the publication of the data.

\section{Competing interests}

The authors declare that they have no competing interests.

\section{References}

1. Losina E, Walensky RP, Geller A, Beddingfield FC III, Wolf LL, Gilchrest BA and Freedberg KA: Visual screening for malignant melanoma: A cost-effectiveness analysis. Arch Dermatol 143: 21-28, 2007.

2. Rigel D: Cutaneous ultraviolet exposure and its relationship to the development of skin cancer. J Am Acad Dermatol 58 (Suppl 2): S129-S132, 2008.

3. Food and Drug Administration: Consumer health information. November 2009. Available at: www.fda.gov/consumer. Accessed Feb 18, 2010.

4. Candido S, Rapisarda V, Marconi A, Malaponte G, Bevelacqua V, Gangemi P, Scalisi A, McCubrey JA, Maestro R, Spandidos DA, et al: Analysis of the B-RafV600E mutation in cutaneous melanoma patients with occupational sun exposure. Oncol Rep 31: 1079-1082, 2014.

5. Gandini S, Sera F, Cattaruzza MS, Pasquini P, Picconi O, Boyle P and Melchi CF: Meta-analysis of risk factors for cutaneous melanoma: II. Sun exposure. Eur J Cancer 41: 45-60, 2005.

6. Elwood JM and Jopson J: Melanoma and sun exposure: An overview of published studies. Int J Cancer 73: 198-203, 1997.

7. Ehrsam E, Kallini JR, Lebas D, Khachemoune A, Modiano P and Cotten H: Fully regressive melanoma - a case without metastasis. J Clin Aesthet Dermatol 9: 42-46, 2016.

8. Smoller BR: Histologic criteria for diagnosing primary cutaneous malignant melanoma. Mod Pathol 19 (Suppl 2): S34-S40, 2006.

9. Zurac S, Negroiu G, Petrescu S, Andrei R, Tebeica T, Popp C, Musţată R, Neagu M, Constantin C, Solovan C, et al: Spectrum of morphologic alterations of regression in cutaneous melanoma potential for improving disease prognosis. Rom J Intern Med 50: 145-153, 2012.

10. Aung PP, Nagarajan P and Prieto VG: Regression in primary cutaneous melanoma: Etiopathogenesis and clinical significance. Lab Invest 97: 657-668, 2017. 
11. Gugger A, Barnhill RL, Seifert B, Dehler S, Moch H, Lugassy C, Marques-Maggio E, Rushing EJ and Mihic-Probst D: Cutaneous melanoma with brain metastasis: Report of 193 patients with new observations. PLoS One 11: e0156115, 2016.

12. Printz C: Spontaneous regression of melanoma may offer insight into cancer immunology. J Natl Cancer Inst 93: 1047-1048, 2001

13. Wenzel J, Bekisch B, Uerlich M, Haller O, Bieber T and Tüting T: Type I interferon-associated recruitment of cytotoxic lymphocytes: A common mechanism in regressive melanocytic lesions. Am J Clin Pathol 124: 37-48, 2005.

14. Håkansson A, Gustafsson B, Krysander L, Hjelmqvist B, Rettrup B and Håkansson L: Expression of ICAM-1 during IFN-alpha-based treatment of metastatic malignant melanoma: Relation to tumor-infiltrating mononuclear cells and regressive tumor changes. J Interferon Cytokine Res 19: 171-177, 1999.

15. Lupu M, Caruntu A, Caruntu C, Papagheorghe LML, Ilie MA, Voiculescu V, Boda D, Constantin C, Tanase C, Sifaki M, et al: Neuroendocrine factors: The missing link in non melanoma skin cancer (Review). Oncol Rep 38: 1327-1340, 2017.

16. Sandru F, Popa A and Dumitrascu MC: Dermoscopic view of vertical growth phase nodular malignant melanoma. Med Image Database 2: 35-36, 2019.

17. Caruntu C, Boda D, Constantin C, Caruntu A and Neagu M: Catecholamines increase in vitro proliferation of murine B16F10 melanoma cells. Acta Endocrinol (Copenh) 10: 545-558, 2014.

18. Boda D: Cellomics as integrative omics for cancer. Curr Proteomics 10: 237-245, 2013.

19. Bories N, Dalle S, Debarbieux S, Balme B, Ronger-Savlé S and Thomas L: Dermoscopy of fully regressive cutaneous melanoma. Br J Dermatol 158: 1224-1229, 2008.
20. Prodea MC, Sandru F, Carpenco E, Gaje PN, Horhat ID, Ceausu AR, Mederle N and Mederle OA: A rare sweat gland tumour-immunohistochemical features. Rev Chim (Bucharest) 70: 1863-1865, 2019.

21. Ilie MA, Caruntu C, Lupu M, Lixandru D, Tampa M, Georgescu SR, Bastian A, Constantin C, Neagu M, Zurac SA, et al: Current and future applications of confocal laser scanning microscopy imaging in skin oncology. Oncol Lett 17: 4102-4111, 2019.

22. Stefan O, Tudor G, Constantinescu C, Luca C, Boda D, Caruntu C, Cioplea M, Nichita L and Zurac SA: E-cadherin and N-cadherin expression pattern in common melanocytic nevi. Virchows Arch 475: S28-S28, 2019.

23. Neagu M,Constantin C, Tanase C and Boda D: Patented biomarker panels in early detection of cancer. Recent Pat Biomark 1: 10-24, 2011.

24. Zurac S, Neagu M, Constantin C, Cioplea M, Nedelcu R, Bastian A, Popp C, Nichita L, Andrei R, Tebeica T, et al: Variations in the expression of TIMP1, TIMP2 and TIMP3 in cutaneous melanoma with regression and their possible function as prognostic predictors. Oncol Lett 11: 3354-3360, 2016.

25. Diaconeasa A, Boda D, Solovan C, Enescu DM, Vîlcea AM and Zurac S: Histopathologic features of Spitzoid lesions in different age groups. Rom J Morphol Embryol 54: 51-62, 2013. 